\title{
A Tightly Coupled BDS/INS Integrated Positioning Algorithm Based on Triple-Frequency Single-Epoch Observations
}

\author{
Fei Ye, Shuguo Pan (D, Wang Gao, Hao Wang, Chun Ma, and Yunfeng Wang \\ School of Instrument Science and Engineering, Southeast University, Nanjing, China \\ Correspondence should be addressed to Shuguo Pan; psg@seu.edu.cn
}

Received 21 November 2020; Revised 24 December 2020; Accepted 16 January 2021; Published 28 January 2021

Academic Editor: Gaoge $\mathrm{Hu}$

Copyright (c) 2021 Fei Ye et al. This is an open access article distributed under the Creative Commons Attribution License, which permits unrestricted use, distribution, and reproduction in any medium, provided the original work is properly cited.

\begin{abstract}
Vehicular dynamic positioning based on tightly coupled (TC) Global Navigation Satellite System (GNSS)/Inertial Navigation System (INS) integration in urban areas is due to either low accuracy of pseudorange or poor continuity of carrier phase, resulting in insufficient positioning performance. To enhance the stability while ensuring positioning accuracy, this paper proposed a tightly coupled Beidou Navigation Satellite System (BDS)/INS integration scheme by improving measurement modelling with triplefrequency observations: first, a stepwise single-epoch ambiguity resolution of extra-wide-lane (EWL)/wide-lane (WL) combined observations and then modelling the measurement equation with fixed WL observation instead of conventional pseudorange or carrier phase. Experiments were carried out for verification with data collected in real traffic by a measurement vehicle. The proposed method achieved single-epoch output with an RMS statistical accuracy of decimetre level of $0.152 \mathrm{~m}$ horizontally and $0.196 \mathrm{~m}$ vertically. The signal outage experiment verified that the proposed algorithm is restoring high-accuracy positioning output in single-epoch once the signal is recaptured. The proposed method obtained a positioning accuracy improvement of $43.6 \%$ horizontally and $6.2 \%$ vertically in signal outage sections compared to the conventional method. This avoids the multiepoch ambiguity searching to fix with conventional carrier-phase processing, thereby improving the positioning stability.
\end{abstract}

\section{Introduction}

With the rapid development of autonomous vehicles, positioning performance in the urban environment has become a hot research point. The tightly coupled (TC) Global Navigation Satellite System (GNSS)/Inertial Navigation System (INS) integration technique is used to provide great absolute positioning output, but the easily blocked satellite signals in an urban environment make the integrated system hard to perform as stably as in an open area. Urban canyons, tunnels, and boulevards covered by thick trees are typical GNSS restrict environments. In these areas, factors such as bad satellite geometric distribution, weak or blocked signal, and even multipath are the most significant error sources which must be considered [1]. Unstable GNSS observations lead to unstable error correction, which forces integrated positioning to more rely on high-end INS performance. Therefore, the current autonomous vehicle environment perception solutions prefer more sensors to perform relative positioning. The problems mentioned above directly lead to a substantial increase in hardware costs and system complexity.

The principle of tightly coupled integration is using two sensors on measuring the same target and then calibrating one of the sensors with the bias measured, to obtain the final output [2]. In the case of GNSS/INS integration, both the GNSS and INS are observing the geometry distance and its changing rate between satellites and the rover station [3]. On the assumption that the GNSS observation value is stable and reliable, the GNSS observations are used to correct the INS measurements to avoid the divergence, thereby achieving calibration [4]. However, GNSS observations in an urban environment cannot guarantee its own stability. In order to improve the positioning performance of the tightly coupled integration, some studies start from the state of vehicle movement in the urban environment. For example, velocity and the small changing altitude are used as constraints for maintaining the INS error bounded in the case of 
GNSS outage [5]. Some scholars improved the fusion algorithm of tightly coupled integration to enhance its robustness and adaptive performance in urban environments $[6,7]$. Besides, researchers also investigated innovative improvements into the environmental constraints, i.e., the context-aided framework [8]. However, the study about GNSS observations' stability improving in complex environments is relatively small.

The most common TC is using pseudorange as GNSS observation [2]. With the technique developed, the pseudorange based meter level accuracy cannot fulfil the requirement of high-accuracy applications. As a higher accuracy observation, carrier phase is first used to smooth pseudorange [9]. Though the accuracy is improved indeed, the real-time performance is reduced. Some studies directly use carrier-phase observation instead $[10,11]$. However, the restriction of using carrier-phase observations is the ambiguity resolution (AR). Generally, the ambiguity resolution requires several continuous epochs to fix. And once the loose lock happened, it must be refixed again. In the case of GNSS/ INS integration, during the refixing period, either use uncontrollable float carrier-phase observations or let INS reckon independently without calibration. The AR process of narrow-lane (NL) carrier phase includes Kalman filter (KF) and LAMBDA (Least-squares Ambiguity Decorrelation Adjustment) algorithm [12]. The serial use of this process and GNSS/INS integrated filtering greatly increases the complexity $[13,14]$.

In order to find a solution that has both simple calculations as using pseudorange and high accuracy as using carrier phase, AR must be processed as simple as possible. Some use the time difference between neighbour epochs [15]. This method can receive a much higher accuracy than using pseudorange. Its error accumulates as time goes on, so this method is only suitable for short-term filtering. Some researchers use wide-lane (WL) observations to form a double-differenced (DD) measurement model and then calculate ambiguity aided by INS [16]. Considering dual frequency, stepwise WL and NL ambiguity resolutions were used for AR in precise point positioning (PPP) [17]. After BeiDou Navigation Satellite System (BDS) started to broadcast triple-frequency signal in 2012, multisystem multifrequency is feasible for a better GNSS positioning model [18] and positioning with triple frequency received wide attention [19]. Using triple-frequency carrier observations can receive a higher AR success rate than using dual frequency [20]. Because extra-wide-lane (EWL) and WL have longer wavelength, AR is realized by rounding to integer [21]. Therefore, EWL/WL technique is able to be used for single-epoch positioning in real-time kinematic (RTK) $[22,23]$. Researchers keep on improving EWL/WL singleepoch AR with triple frequency based on the geometry-free and ionosphere-free (GIF) model and three-carrier ambiguity resolution (TCAR) method [24-28]. Besides, positioning with triple frequency allows not only avoiding but also detecting and repairing cycle slip [29]. Thus, EWL/WL based single-epoch positioning is ideal for improving continuity while ensuring accuracy to decimetre or subdecimetre level [22].
The research we are working on aims to use the excellent performance of BDS to achieve stable high-accuracy vehicular positioning in urban environments. In this paper, we improved TC measurement modelling with three-frequency single-epoch AR technology and BDS observations. With the proposed method, on the one hand, INS is calibrated with ambiguity fixed observations once a satellite signal is observable, thereby improving the positioning accuracy and continuity. On the other hand, due to the independence of epochs, the interference will not pollute the output of neighbour epochs, thereby improving the positioning stability.

The rest of the paper is organized as follows. In Section 2, based on the BDS triple-frequency single-epoch observations, a tightly coupled BDS/INS integration with WL observation is proposed, and the corresponding integration architecture is given. In order to verify the effectiveness and advantages of the proposed method, a series of experiments are carried out in Section 3, together with detailed experimental procedures, and results are given. Section 4 analyses the experimental results in detail and discusses the advantages and disadvantages of the proposed method. The conclusion and future work are given in Section 5 .

\section{Materials and Methods}

2.1. BDS Triple-Frequency Single-Epoch Observations. Triple frequency of pseudorange and carrier-phase linear combinations is formed first. Then, the EWL fixed carrier phase is obtained by the geometric-free ionosphere-free model. With help of the fixed EWL observation and TCAR algorithm, the WL ambiguity is fixed by rounding to integer; thus, the accurate WL observation is obtained. The specific progress in detail is as follows.

EWL and WL are different linear combinations of observations. The combined observations have longer wavelengths, which are easier to fix the ambiguity. EWL has a longer wavelength than WL. For BDS observations, the combination coefficient of EWL is $(0,-1,1)$ and for WL is $(1,-1,0)$ [30]. The linear combinations are formed following the equations:

$$
\begin{aligned}
& \Delta \nabla \phi_{(i, j, k)}=\frac{i f_{1} \Delta \nabla \phi_{1}+j f_{2} \Delta \nabla \phi_{2}+k f_{3} \Delta \nabla \phi_{3}}{i f_{1}+j f_{2}+k f_{3}}, \\
& \Delta \nabla P_{(i, j, k)}=\frac{i f_{1} \Delta \nabla P_{1}+j f_{2} \Delta \nabla P_{2}+k f_{3} \Delta \nabla P_{3}}{i f_{1}+j f_{2}+k f_{3}},
\end{aligned}
$$

where $f_{1}, f_{2}$, and $f_{3}$ are three frequencies. $i, j$, and $k$ are the combination coefficients. $\Delta \nabla$ represents the double-differenced operator. $\phi_{n}$ and $P_{n}$ are the carrier-phase and pseudorange observations of the $n$-th frequency in the unit of meter. The geometry-free and ionosphere-free (GIF) model is used to calculate the ambiguity parameter of EWL [31]. After double difference between stations and satellites in the short baseline, the ionospheric error was eliminated. The calculation is shown as follows: 


$$
\Delta \nabla N_{\mathrm{EWL}}=\left[\frac{\Delta \nabla \phi_{\mathrm{EWL}}-\Delta \nabla P_{\mathrm{EWL}}}{\lambda_{\mathrm{EWL}}}\right],
$$

where $\lambda_{\text {EWL }}$ is the wavelength of EWL observation and the $\Delta \nabla N_{\text {EWL }}$ is the EWL ambiguity, which is fixed by rounding to integer. The fixed EWL ambiguity will be used as a known value to participate in the calculation of WL ambiguity based on the TCAR method as follows:

$$
\begin{aligned}
\Delta \nabla N_{\mathrm{WL}}= & \frac{1}{\lambda_{\mathrm{WL}}}\left[\Delta \nabla \phi_{\mathrm{WL}}-\Delta \nabla \phi_{\mathrm{EWL}}-\left(\eta_{\mathrm{EWL}}-\eta_{\mathrm{WL}}\right) \Delta \nabla I\right. \\
& \left.+\lambda_{\mathrm{EWL}} \Delta \nabla N_{\mathrm{EWL}}\right],
\end{aligned}
$$

where $\lambda_{\mathrm{WL}}$ is the wavelength of WL observation and $\Delta \nabla N_{\mathrm{WL}}$ is the WL ambiguity. $I$ is the ionospheric delay, together with the coefficient $\eta$, the influence of which is controllable. Same as $\Delta \nabla N_{\mathrm{EWL}}, \Delta \nabla N_{\mathrm{WL}}$ is also rounding to fix. Once the ambiguity of WL is fixed, the carrier-phase observation is also fixed:

$$
\Delta \nabla \phi_{\mathrm{WL}}=\Delta \nabla \rho+\Delta \nabla T-\eta_{\mathrm{WL}} \Delta \nabla I+\lambda_{\mathrm{WL}} \Delta \nabla N_{\mathrm{WL}}+\Delta \nabla \varepsilon_{\mathrm{WL}},
$$

where $T, I$, and $\varepsilon$ are received by interpolation of area modelling of network RTK. Constants as $\eta_{\mathrm{EWL}}=-0.352$ and $\lambda_{\mathrm{WL}}=0.8470 \mathrm{~m}$ are given in Gao's research [31]. The only unknown parameter is the double-differenced satellite-station geometry distance $\Delta \nabla \rho$. For dynamic application, $\Delta \nabla \rho$ is used for modelling the measurement equation of the tightly coupled integration.

\subsection{Tightly Coupled BDS/INS Integration}

2.2.1. System Model. The system model of tightly coupled integration system is established in the following form [32]:

$$
\dot{x}(t)=f(x(t))+w(t),
$$

where $f(\cdot)$ is the nonlinear function of system dynamics with state $x(t)$ and $w(t)$ is the system noise vector. The discrete form of (5) is described as follows:

$$
X_{k}=\Phi_{k, k-1} X_{k-1}+W_{k-1},
$$

where $X_{k}$ is the estimated state vector in the kth epoch, while $X_{k-1}$ is the state vector of the last epoch; $\Phi_{k, k-1}$ is the discrete form of $f(\bullet)$; and $W_{k-1}$ is the discrete version of system noise vector. The system modelling is derived from the dynamic equation of INS $[33,34]$.

Since the double-differenced observations do not need to estimate the satellite clock bias, the observable errors are only INS errors. Considering ECEF-frame, the state vector is selected as the most basic 15-state solution, which is

$$
X=\left\{\begin{array}{lllll}
\delta r_{1 \times 3} & \delta v_{1 \times 3} & \varphi_{1 \times 3} & b_{g, 1 \times 3} & b_{a, 1 \times 3}
\end{array}\right\}^{T},
$$

where $\delta r$ stands for positioning error vector in three dimensions. Similar, $\delta v$ and $\varphi$ stand for velocity and attitude error vectors. $b_{g}$ and $b_{a}$ are measurement drift/bias vectors of gyros and accelerometers. With the state vector determined, the system error dynamics of GNSS/INS integration is so that becomes basically an INS mechanization equation. It is written in ECEF-frame according to [34] as

$$
\begin{aligned}
{\left[\begin{array}{c}
\delta \dot{r}^{e} \\
\delta \dot{v}^{e} \\
\dot{\varphi}^{e}
\end{array}\right]=} & {\left[\delta v^{e}+\varepsilon_{r}^{e}-2 \Omega_{i e}^{e} \times \delta v^{e}+C_{b}^{e} f^{b} \varphi^{e}+C_{b}^{e} b_{a}^{b}+\varepsilon_{v}^{e}\right.} \\
& \left.-\Omega_{i e}^{e} \times \varphi^{e}+C_{b}^{e} b_{g}^{b}+\varepsilon_{\varphi}^{e}\right],
\end{aligned}
$$

where $\Omega_{i e}$ is the skew-symmetric form of the Earth rotation rates and $C_{b}^{e}$ represents the rotation matrix from base-frame to ECEF, here to rotate the specific force $f^{b}$ to ECEF. $\varepsilon_{r}, \varepsilon_{v}$, and $\varepsilon_{\varphi}$ indicate random walk process driving noise vectors for the position, velocity, and attitude, respectively.

Initially, the GNSS/INS integrated positioning algorithm was designed for highly dynamic aircraft, and the dynamics of the vehicular platform (such as autonomous vehicles) is not as complicated as that of aircraft. Generally, in vehicular applications, the parameters such as height errors and threedimensional acceleration do not change frequently and greatly, so the detailed modelling with those parameters can be simplified to a certain extent in practical applications. For example, it is also mentioned in [5] that the velocity of the vehicular platform in the lateral and elevation directions can be almost assumed to be zero. Besides, if low-cost MEMS IMU is used and the instrumental error of the gyro itself is far greater than the influence of other biases, then only $\varepsilon_{\varphi}^{e}$ needs to be retained for the estimation of the attitude error. When the simplified system model participates in the estimation, the computation can be further reduced and lay a foundation for the feasibility of autonomous vehicle mass applications.

Finally, the system model is presented based on the system error dynamics as follows:

$$
\left[\begin{array}{c}
\delta \dot{r} \\
\delta \dot{v} \\
\dot{\varphi} \\
\dot{b}_{g} \\
\dot{b}_{a}
\end{array}\right]=\left[\begin{array}{ccccc}
0 & I & 0 & 0 & 0 \\
0 & -2 \Omega_{i e} & C_{b}^{e} f^{b} & 0 & C_{b}^{e} \\
0 & 0 & -\Omega_{i e} & C_{b}^{e} & 0 \\
0 & 0 & 0 & 0 & 0 \\
0 & 0 & 0 & 0 & 0
\end{array}\right] \cdot\left[\begin{array}{c}
\delta r \\
\delta v \\
\varphi \\
b_{g} \\
b_{a}
\end{array}\right]+\left[\begin{array}{c}
\varepsilon_{r} \\
\varepsilon_{v} \\
\varepsilon_{\varphi} \\
0 \\
0
\end{array}\right] .
$$

2.2.2. Measurement Model. Similar with the system model, the measurement model of integrated positioning system describes the relationship between observation and the estimated states. The origin measurement model is normally formed as follows:

$$
Z_{k}=H_{k} X_{k}+V_{k},
$$

where $Z_{k}$ represents the measurement vector at epoch $k$. $H_{k}$ is the measurement mapping matrix, which expresses relationship between states and measurement vectors. $V_{k}$ denotes measurement error. Normally, measurement vector $Z_{k}$ in the TC model reflects the measurement difference of two sensors on the same object. Geometry distance is the 
most common selection. In case of this paper, measurement vector is expressed as follows:

$$
Z_{k}=\Delta \nabla \rho_{G, k}-\Delta \nabla \rho_{I, k}
$$

where $\Delta \nabla \rho_{G, k}$ and $\Delta \nabla \rho_{I, k}$ are representing DD geometry distance measured by GNSS and INS. Geometry distance between satellite and INS approximate position is used to form $\rho_{I}$. The BDS triple-frequency WL observation is used to form $\rho_{G}$ instead of conventional pseudorange or carrierphase observations. Therefore, in the rest of the section, we use $\rho_{\mathrm{WL}}$ to represent the observation.

In the measurement equation, geometry distance received by two systems is used for modelling $H_{k}$. Once $\Delta \nabla \rho_{\mathrm{WL}}$ and $\Delta \nabla \rho_{I}$ received, the error between the two can be described by the equation as follows:

$$
\begin{aligned}
\Delta \nabla \rho_{W L}-\Delta \nabla \rho_{I}= & \left(\Delta \nabla l_{G}-\Delta \nabla l_{I}\right) \delta x+\left(\Delta \nabla m_{G}-\Delta \nabla m_{I}\right) \delta y \\
& +\left(\Delta \nabla n_{G}-\Delta \nabla n_{I}\right) \delta z+\varepsilon
\end{aligned}
$$

where $\Delta \nabla l, \Delta \nabla m$, and $\Delta \nabla n$ represent DD direction cosine. $\delta x, \delta y$, and $\delta z$ mean positioning error between GNSS and INS. Subscripts $G$ and $I$ represent values of GNSS and INS. Let $e$ represent the direction cosines for short, such as

$$
\Delta \nabla e_{k}=\left[\Delta \nabla l_{G, k}-\Delta \nabla l_{I, k} \quad \Delta \nabla m_{G, k}-\Delta \nabla m_{I, k} \quad \Delta \nabla n_{G, k}-\Delta \nabla n_{I, k}\right] .
$$

Then, considering other error states, there should be an $n \times 15$-dimensional measurement matrix to describe relationship between $Z_{k}$ and $\widehat{X}_{k}$ (the estimated state). $n$ stands for satellite numbers in the epoch involved. Then, $H_{k}$ is written as follows:

$$
H_{k}=\left[\begin{array}{ll}
\Delta \nabla e_{k, n \times 12} & 0_{1 \times 12}
\end{array}\right] .
$$

The DD eliminates clock error between satellite and receiver; thus, the equivalent distance caused by clock error is no longer involved. Finally, the measurement equation is written as follows:

$$
\left[\begin{array}{c}
\Delta \nabla \rho_{W L, k}^{1}-\Delta \nabla \rho_{I, k}^{1} \\
\Delta \nabla \rho_{W L, k}^{2}-\Delta \nabla \rho_{I, k}^{2} \\
\vdots \\
\Delta \nabla \rho_{W L, k}^{n}-\Delta \nabla \rho_{I, k}^{n}
\end{array}\right]=\left[\begin{array}{cc}
\Delta \nabla e_{1, k} & 0_{1 \times 12} \\
\Delta \nabla e_{2, k} & 0_{1 \times 12} \\
\vdots & \\
\Delta \nabla e_{n, k} & 0_{1 \times 12}
\end{array}\right] \cdot X+\left[\begin{array}{c}
\varepsilon_{1, k} \\
\varepsilon_{2, k} \\
\vdots \\
\varepsilon_{n, k}
\end{array}\right] .
$$

2.2.3. Integration Scheme. In GNSS/INS integration, with the usage of conventional carrier-phase observations, it is necessary to use a first KF for float ambiguity estimation and LAMBDA for fixed ambiguity searching; then, the second KF for GNSS/INS fusion can be performed. Or, in another solution, the ambiguity is appended to the state vector and estimated together. Although double KF usage is avoided, the increased ambiguity parameter greatly increases the matrix dimension and also the corresponding computation pressure. Therefore, in the case when the conventional carrier-phase observation processing is used, these two schemes have both shortcomings for an autonomous vehicle to use. With the proposed algorithm, the ambiguity is fixed in a single epoch. The benefits of high-accuracy positioning and less computation are both covered. Figure 1 presents the schematic diagram. The meanings of the signs used in the diagram are the same as those in the model explanation in the rest of the sections.

Cubature Kalman filter (CKF) [35] is the most popular fusion estimator for the GNSS/INS integration in recent years. Compared with the conventional KF variants, CKF has higher accuracy and less computation. As presented in the architecture, the fusion algorithm we used for BDS/INS integration is CKF. Estimation algorithms based on Gaussian filtering generally require state and measurement modelling, and the CKF is no exception. Figure 2 presents the flowchart of CKF.

\section{Results}

3.1. Experiment Setup. The vehicular test was carried out with a measurement vehicle (see Figure 3 ). In the trunk of the vehicle, a set of reference systems was assembled. The reference system was set up with a whole NovAtel SPAN system with an ISA-100C IMU and a PP6 GNSS receiver (Figure 4). The reference system was used for evaluating the performance of the developed tightly coupled BDS/INS integrated positioning system. In addition, a GNSS receiver (Trimble BD990) and a microelectromechanical system (MEMS) INS (XW-5651) are deployed for applying the proposed algorithm (Figure 5). The receiver is specifically receiving BDS triple-frequency observations. Both reference and BDS receiver are connected with the same antenna with a signal splitter, to make sure they are processing the same signal. The specifics of IMUs used in the testing are listed in Table 1.

During the vehicular field test, both the reference and developed systems are connected to continuous operating reference stations (CORS) network. Reference system using data was provided by CORS for high accuracy after positioning. The test system uses one of the base stations to form short-baseline double-difference observation. The offline process software Waypoint Inertial Explorer (IE) is used for processing high-accuracy positioning results. The test environment is chosen in a relatively open area with real traffic. In this test, the vehicle is driven at a normal traffic speed of $30-60 \mathrm{~km} / \mathrm{h}$.

3.2. Field Test. To test the positioning performance of the proposed algorithm, real observations of BDS are collected with help of the measurement vehicle and the reference stations. 2400 epochs of short-baseline differential data with triple-frequency BDS observations were involved in the calculation. The driving trajectory is shown in Figure 6. 


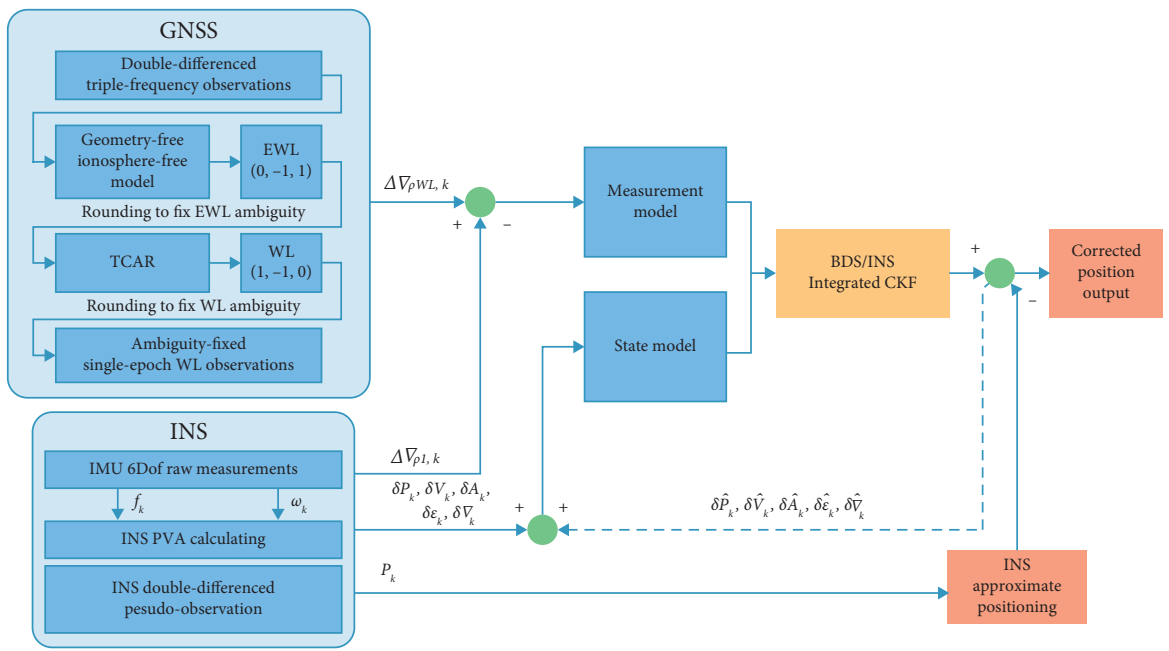

FIgURE 1: The proposed integration scheme.

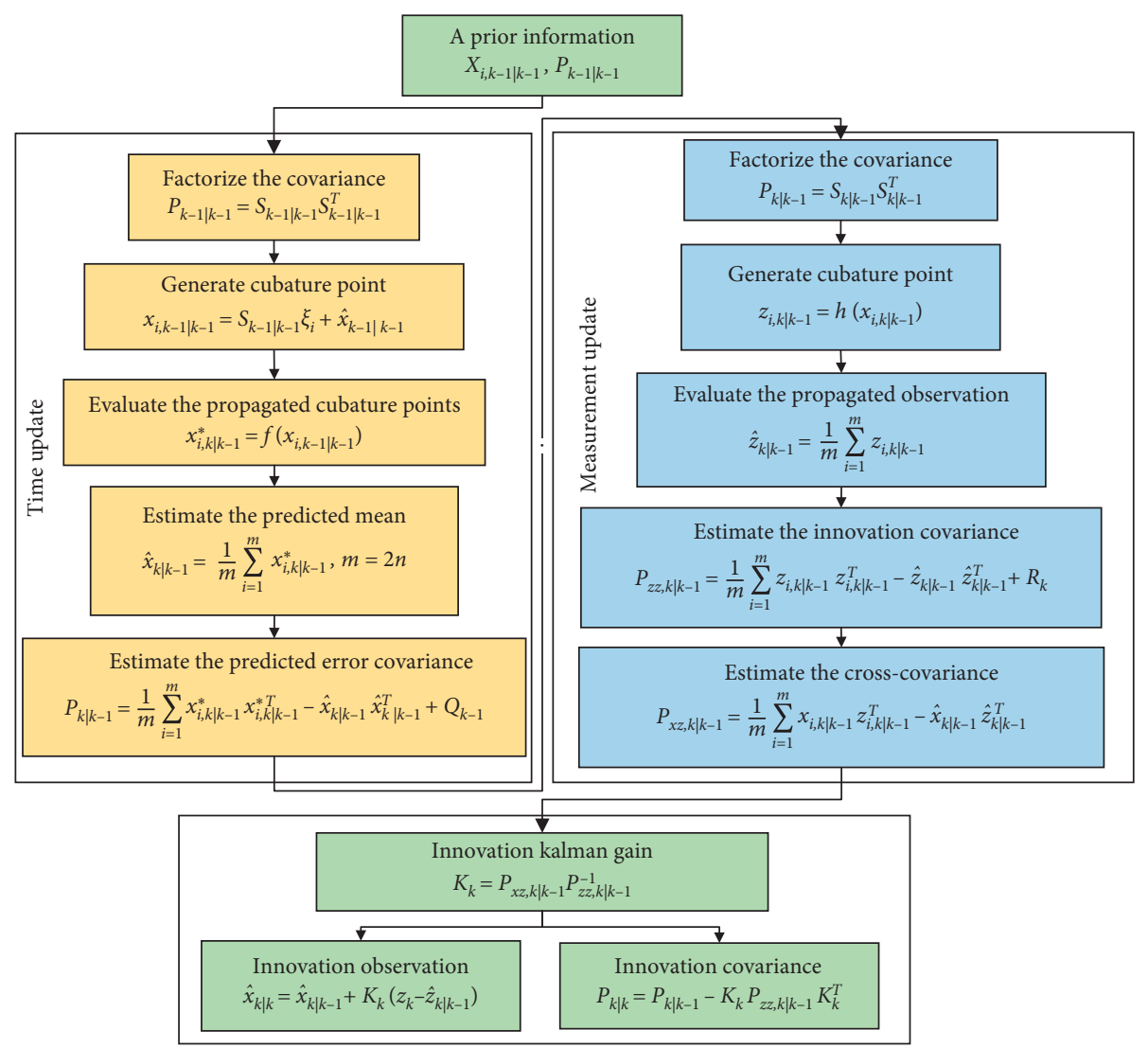

Figure 2: The flowchart of CKF.

Positioning error is obtained by the difference between process output and ground truth value. The corresponding visible satellite numbers are shown in Figure 7 . The errors of all epochs are plotted in Figure 8. The 95\% empirical confidence which is shown in Figure 9 is presenting the reliability of the proposed algorithm. The skyplot for one of the epochs is presented in Figure 10. The accuracy represented with RMS statistics is given in Table 2 .

3.3. Signal Outage Test. A signal outage experiment is designed by artificially removing satellite signals in the 


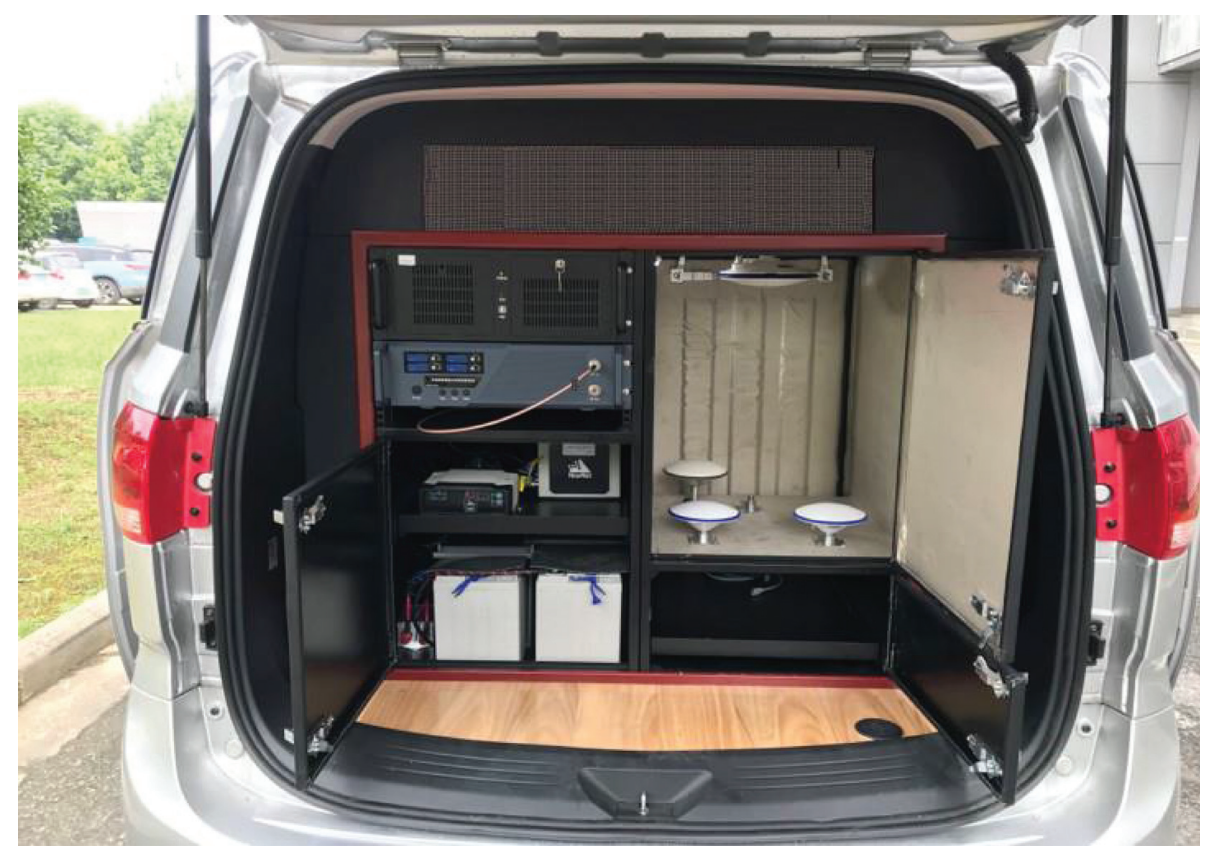

Figure 3: The measurement vehicle for testing.

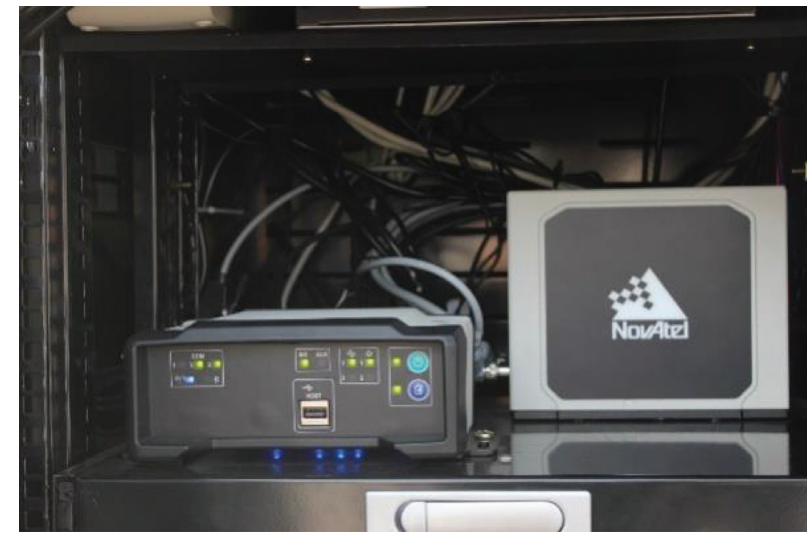

Figure 4: NovAtel SPAN-ISA-100C system.

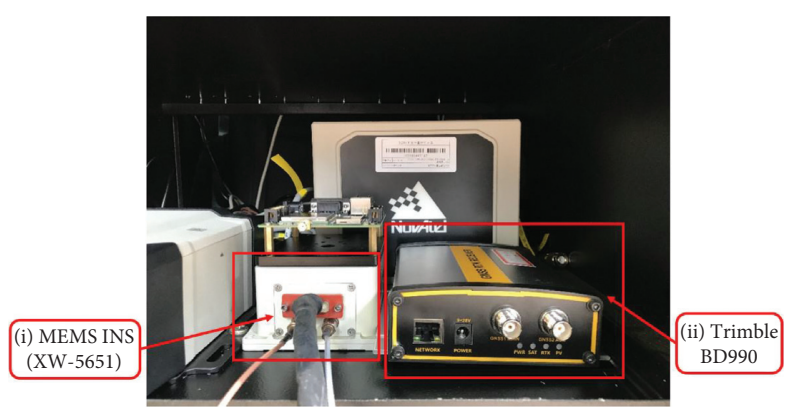

FIgURE 5: The MEMS INS and BDS receiver for verifying the proposed algorithm. (a) The MEMS INS XW-5651. (b) The GNSS receiver Trimble BD990. 
Table 1: Specifications of IMUs.

\begin{tabular}{lcccc}
\hline Items & & ISA-100C & & XW-5651 \\
& Gyroscope & Accelerometer & Gyroscope & Accelerometer \\
\hline Bias & $0.5 \mathrm{deg} / \mathrm{hr}$ & $1250 \mu \mathrm{g}$ & $6 \mathrm{deg} / \mathrm{hr}$ & $1400 \mathrm{ppm}$ \\
Scale factor & $100 \mathrm{ppm}$ & $100 \mathrm{ppm}$ & $1500 \mathrm{ppm}$ \\
Random walk & $0.3 \mathrm{deg} / \sqrt{\mathrm{hr}}$ & $100 \mu \mathrm{g} / \sqrt{\mathrm{hr}}$ & $0.3 \mathrm{deg} / \sqrt{\mathrm{hr}}$ & $0.25 \mathrm{mg} / \sqrt{\mathrm{hr}}$ \\
\hline
\end{tabular}

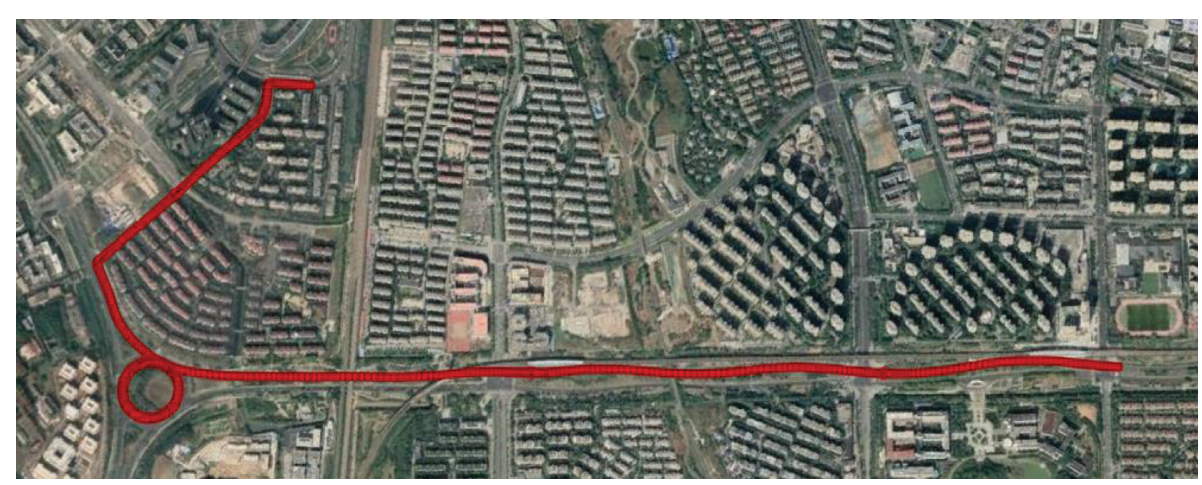

Figure 6: Vehicle trajectory.

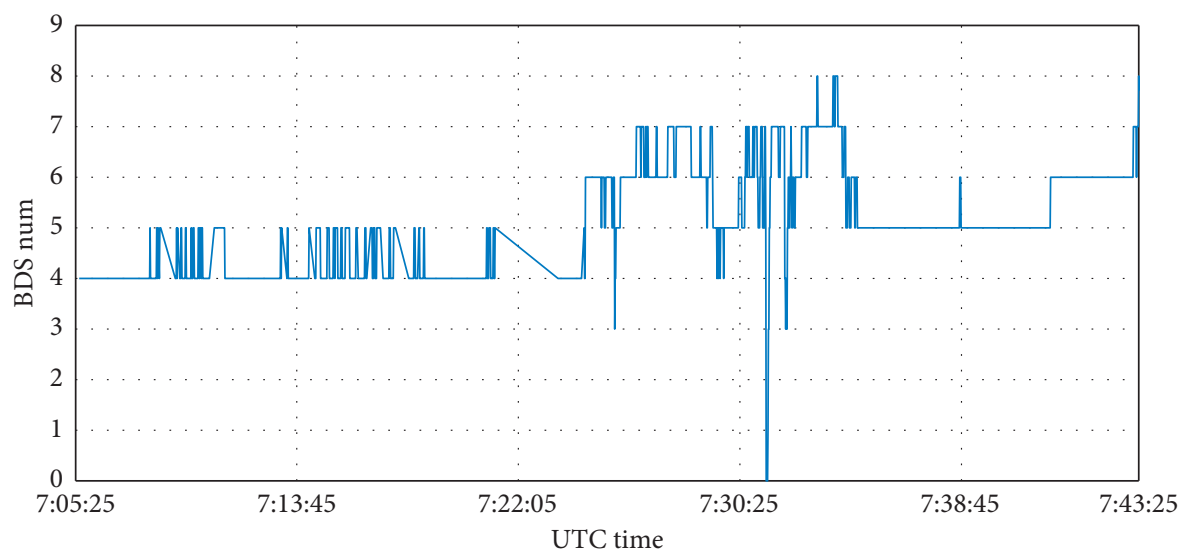

FIgURE 7: Observable satellite number.
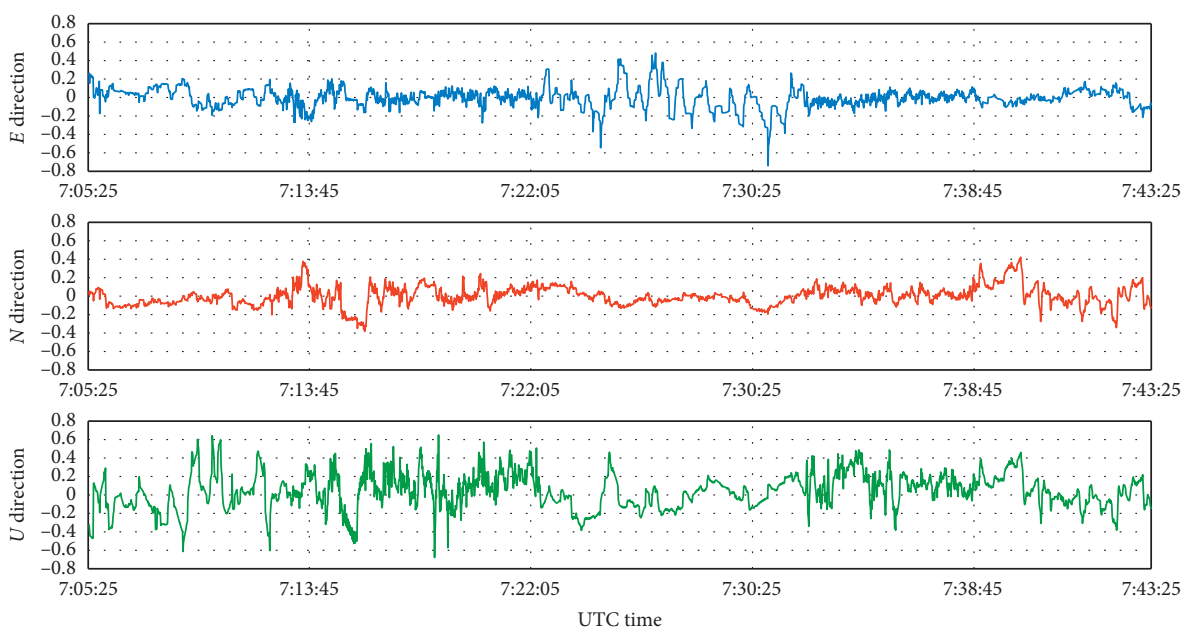

FIgURE 8: Positioning error of proposed TC algorithm based on triple frequency (m). 


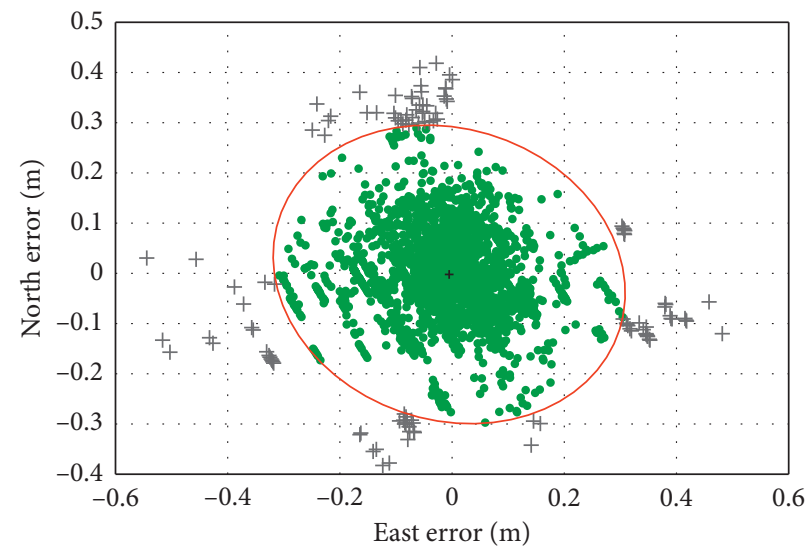

- Dots inside

+ Dots outside

_ Confidence ellipse

(a)

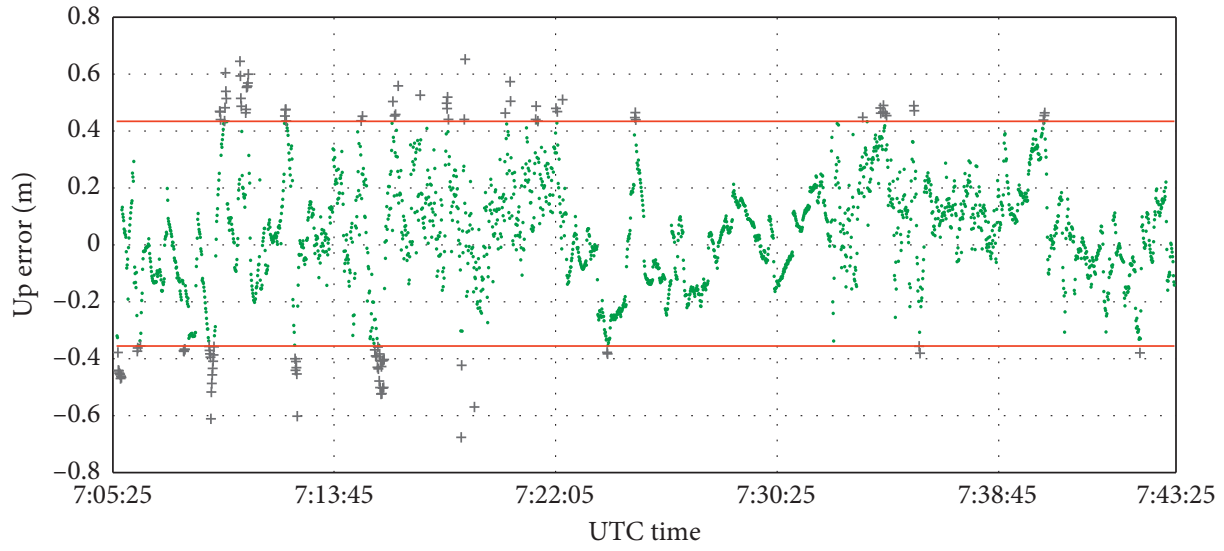

Dots inside

$+\quad$ Dots outside

- Confidence interval

(b)

Figure 9: The 95\% empirical (red) confidence ellipses/intervals for (a) horizontal (N, E) position scatter and corresponding and (b) vertical (U) time series for the proposed model (green for inside and gray for outside).

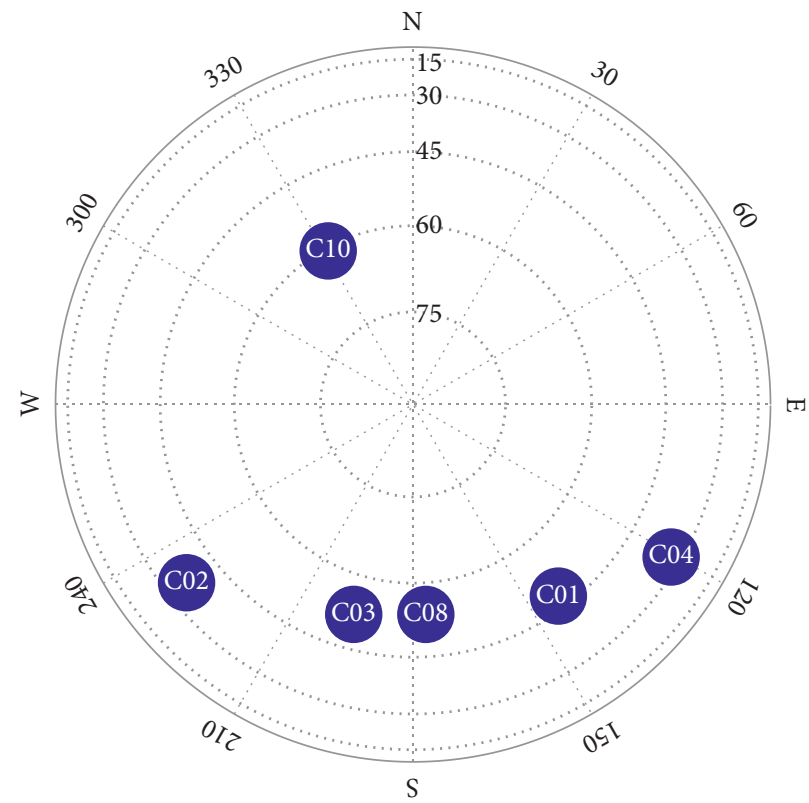

Figure 10: Skyplot of one epoch. 
experimental traffic section. Two lengths of common time interval used in inertial navigation experiments are designed in this experiment, where the signal outage durations of $10 \mathrm{~s}$ and $30 \mathrm{~s}$ were selected. The outages are designed as shown in Figure 11.

To verify the relationship between positioning results and visible satellite numbers, Figures 12 and 13 show changes of visible satellites with triple-frequency observations and positioning error at the same time. The outage of visible satellite numbers in the figure is obtained by removing satellite observations of the corresponding epoch during data processing. The simulated blockage is easier to control the time length of outages. We focused on the positioning error curve around the outages. The positioning error and the corresponding satellite numbers are presented accordingly. The set of figures also includes the effect comparison of the proposed integration algorithm based on triple-frequency WL single-epoch observations versus the conventional algorithm. The conventional method refers to the method that the GNSS observation comes from the continuous measurement of multiple epochs. The float ambiguity is first estimated with KF and then fixed with LAMBDA.

As presented in Figures 12 and 13, the proposed algorithm instantly restores stable positioning output once the satellite signals are restored, while the conventional algorithm delays 4-5 epochs before returning to the stable output. Within the epochs, the divergence stopped under the effect of float ambiguity. But because the float ambiguity is uncontrollable, the positioning result is still not stable.

We have counted the average statistical errors of three outages with the same length, as indicated in Table 3 for $10 \mathrm{~s}$ outages. The 15 epochs before and after each outage were involved in statistics. In the table, the statistics of RMS, mean value, and maximum deviation range of the two methods are, respectively, carried out.

The average statistical errors of three $30 \mathrm{~s}$ outages are presented in Table 4.

Compared with the conventional method, the positioning accuracy of the proposed method improves $43.6 \%$ horizontally and $6.2 \%$ vertically under the $10 \mathrm{~s}$ outages, with $27.5 \%$ in horizontal and $7.4 \%$ under 30 s outages (Table 5 ).

\section{Discussion}

The first experiment is the functional verification of the proposed method. It can be clearly seen from the satellite number that the satellite is being frequently changed or blocked. It is obvious that the frequent satellite shifting does not cause obvious interference on the positioning outputs. The statistical results in the table demonstrate that the proposed method achieves a promising sub-decimetre-level positioning accuracy in an environment with frequent switches of observable satellites, which also meets the theoretical expectations brought by the ambiguity fixed WL observations. With help of the $95 \%$ confidence statistical
TABLE 2: Statistical positioning accuracy.

\begin{tabular}{lc}
\hline Directions & RMS (m) \\
\hline E & 0.110 \\
$\mathrm{~N}$ & 0.104 \\
$\mathrm{U}$ & 0.196 \\
Horizontal & 0.152 \\
\hline
\end{tabular}

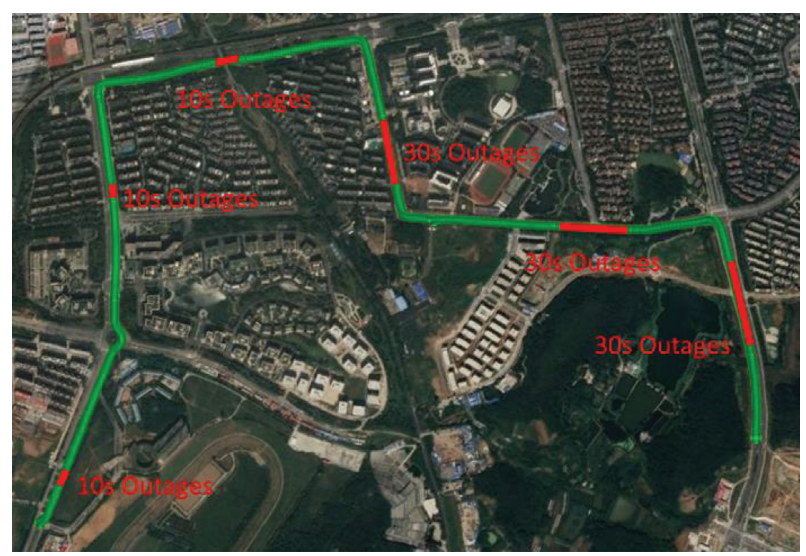

Figure 11: BDS signal outage arrangements.

information, it is proved that, with the proposed tightly coupled triple-frequency BDS/INS integration, a reliable and stable subdecimetre positioning accuracy is achieved when driving in a traffic environment with vehicular dynamic.

The second experiment is about the verification of positioning recovery after signal interruption. The strong competitiveness of the proposed method is when operating in urban areas where signals are easily blocked. As shown in Figures 12 and 13, the conventional method performs better with sufficient satellite observable because of the NL ambiguity fixed with the LAMBDA algorithm. However, when the satellite visibility recovers from blockage, because of the multiepoch ambiguity fixing requirement, a delay of several epochs appeared before stable output was obtainable. In those epochs, although the uncontrollable float solution prevented the positioning error from continuing to diverge, the positioning result was not stable. After the ambiguity is finally fixed, the stable positioning output is recovered. In contrast, the algorithm proposed in this paper fixes the ambiguity at the moment the satellite resumes observable. The stable positioning output is restored at the first epoch that the satellite observation restored, and therefore, relatively stable positioning performance is obtained under signal outages. Such an improvement has also been proved in the statistical tables.

It is worth noting that although the proposed algorithm has achieved the expected performance, an important prerequisite for achieving a single-epoch ambiguity fixing is a short baseline (less than $10 \mathrm{~km}$ ). For a longer baseline, the impact of atmospheric errors must be considered. 


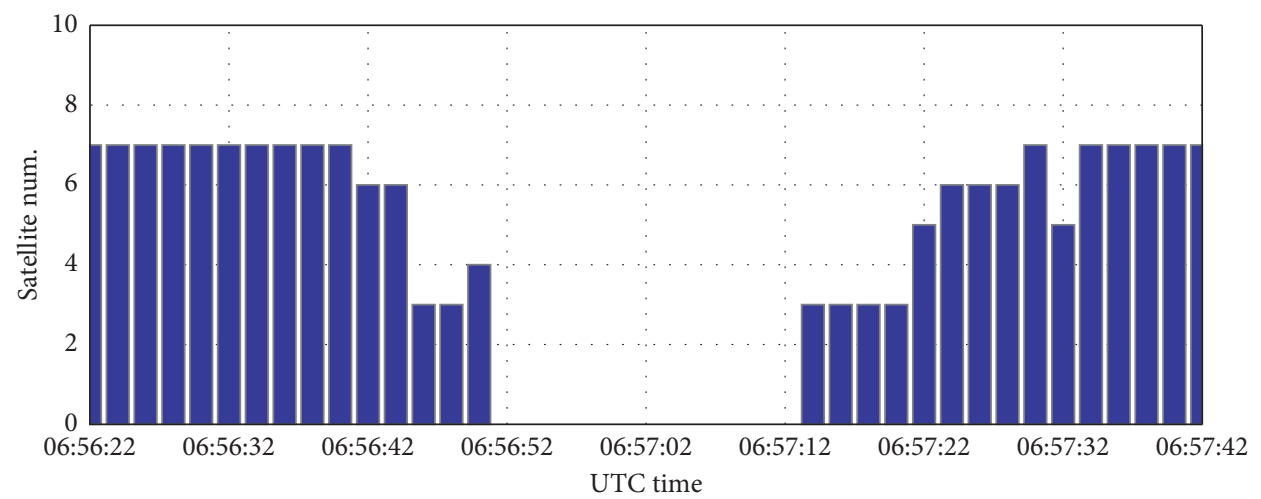

(a)

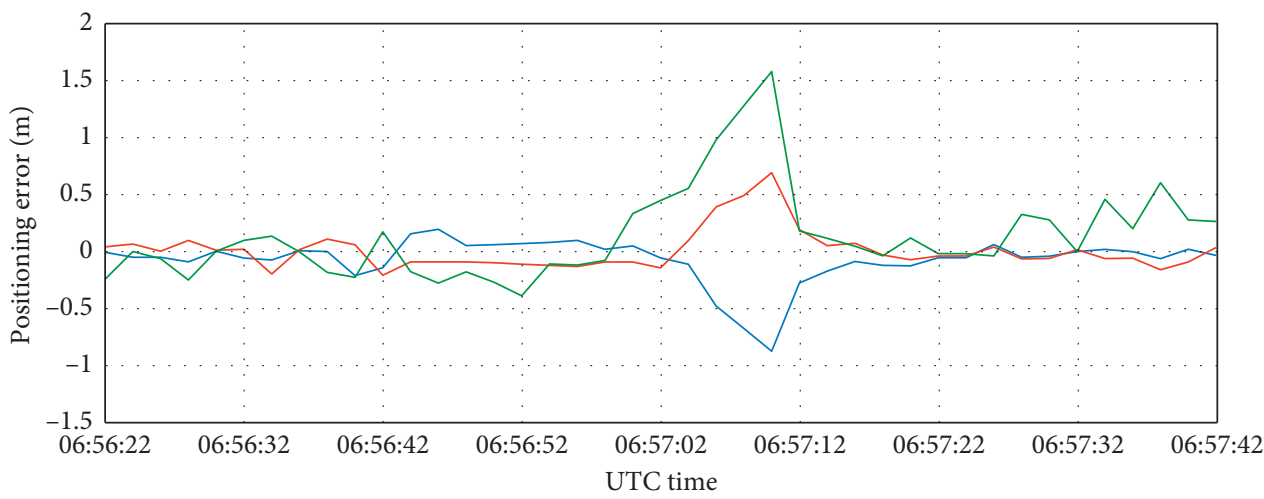

- E

$-\mathrm{N}$

$-\mathrm{U}$

(b)

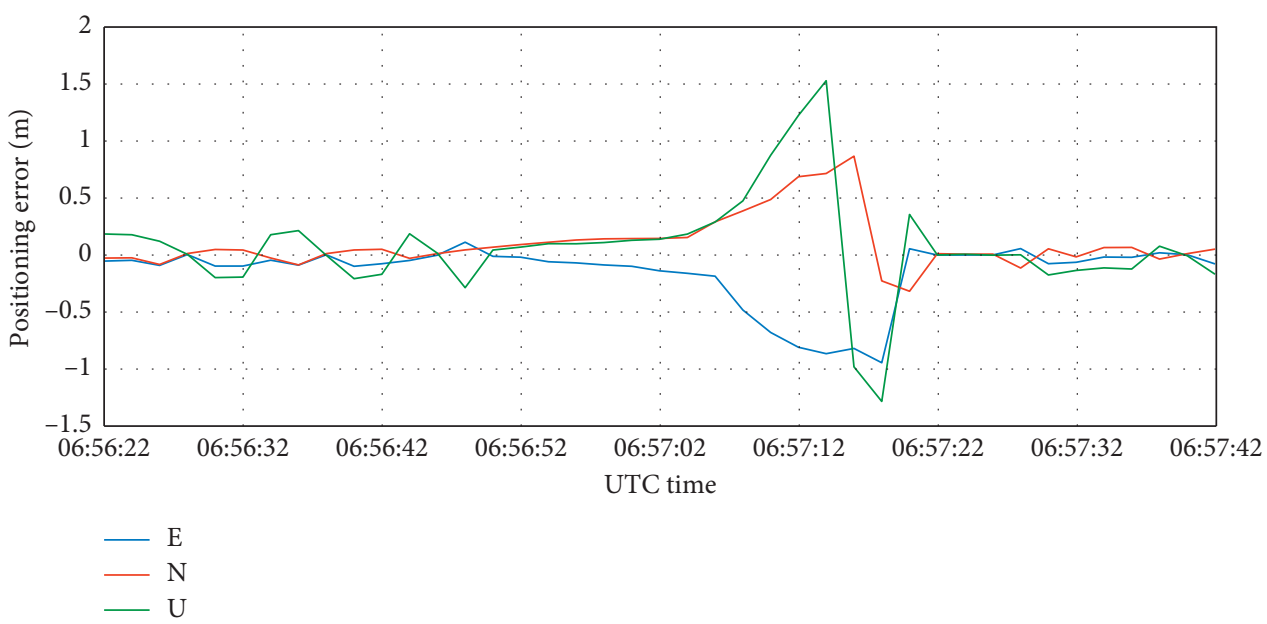

(c)

FIGURE 12: Positioning errors of 10 s outage compared with visible satellite numbers. (a) The corresponding visible satellite numbers. (b) The positioning error curve obtained with the proposed method. (c) The positioning error curve obtained with the conventional method.

\section{Conclusions}

This paper contributed improved tightly coupled BDS/INS integrated positioning utilizing BDS's triple-frequency observation characteristics. By introducing a single-epoch ambiguity resolution technique, a measurement modelling based on BDS triple frequency is proposed, which realizes an improvement of tightly coupled integration. This method enables BDS/INS integrated positioning algorithm to have both a simple scheme and high positioning accuracy in 


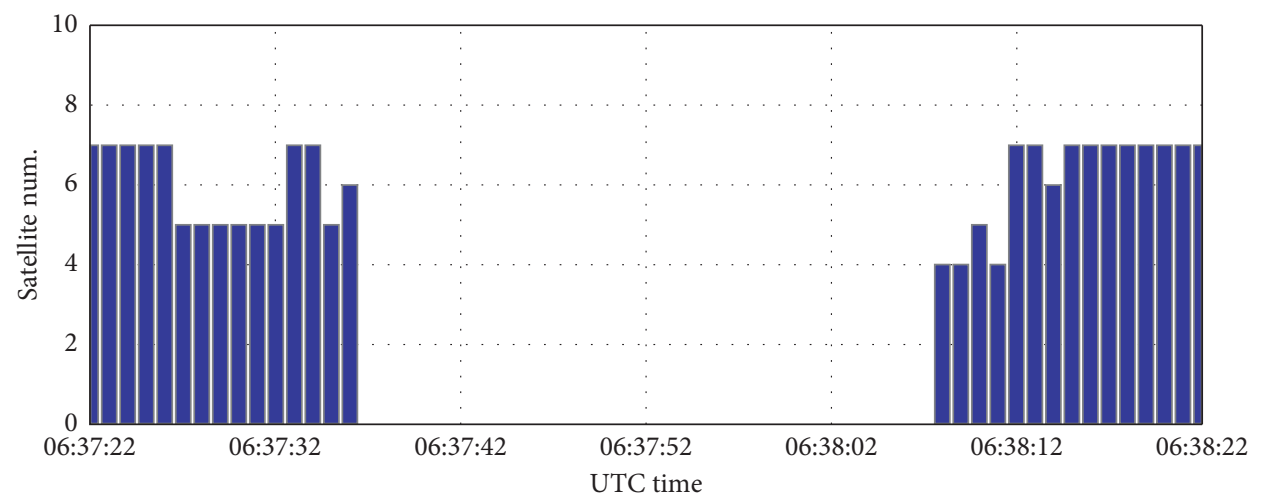

(a)

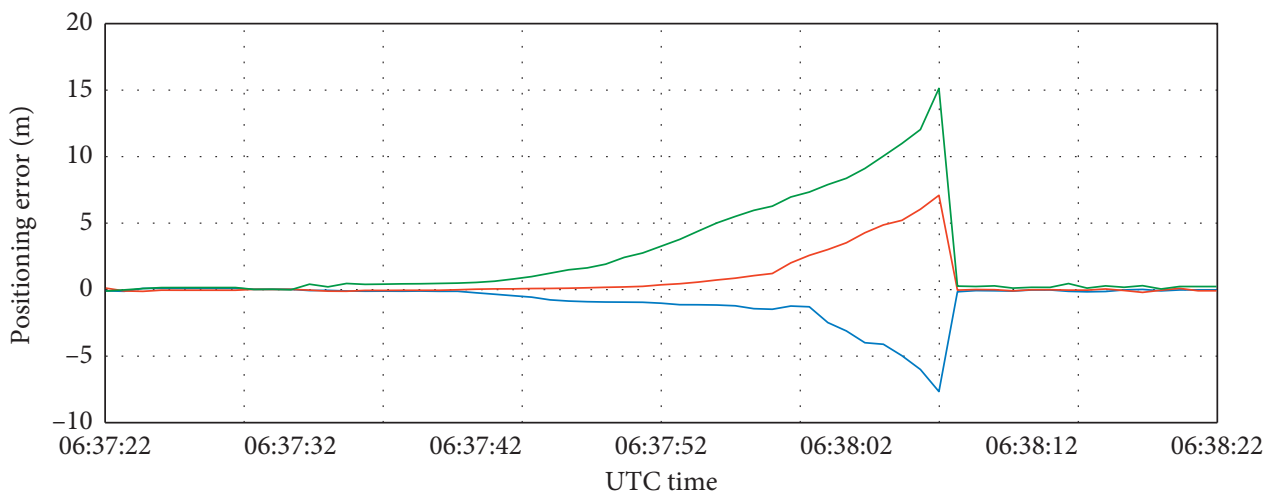

- E

- N

$-\mathrm{U}$

(b)

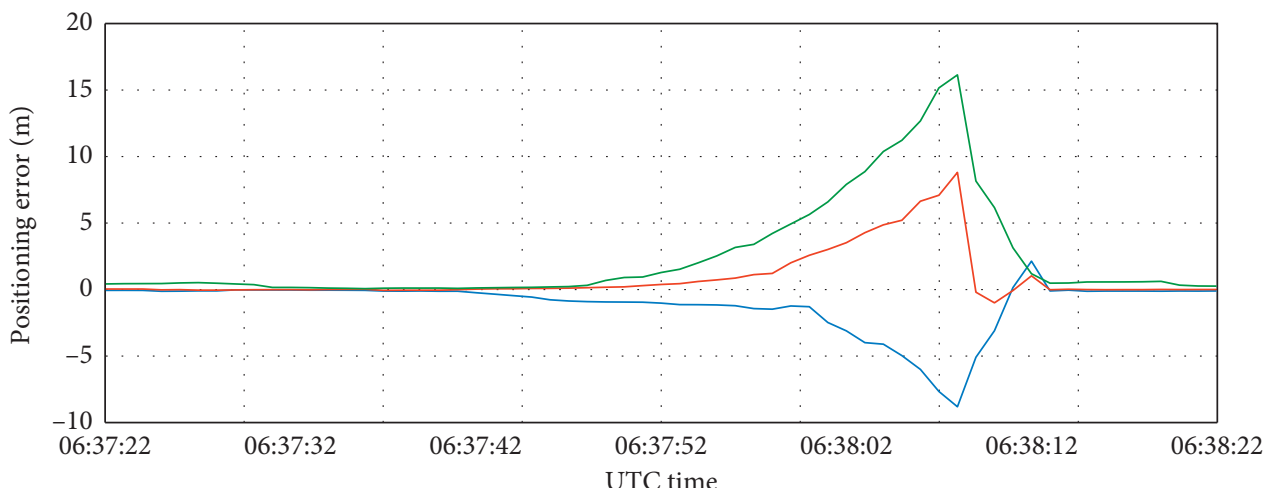

- E

$-\mathrm{N}$

$-\mathrm{U}$

(c)

FIGURE 13: Positioning errors of 30 s outage compared with visible satellite numbers. (a) The corresponding visible satellite numbers. (b) The positioning error curve obtained with the proposed method. (c) The positioning error curve obtained with the conventional method.

urban areas. As a result of the vehicular test in an actual traffic environment accompanied by frequent satellite varying, the positioning accuracy is stable and achieves $0.152 \mathrm{~m}$ for horizontal and $0.196 \mathrm{~m}$ for vertical positioning. The results prove that the proposed algorithm is reliable and meets the theoretical prospect of stable decimetre level positioning accuracy. The ability to restore stable positioning output in a single epoch was verified in the signal outage experiment. Compared with the conventional method, the positioning accuracy of the proposed method improves $43.6 \%$ horizontally and $6.2 \%$ vertically under the 10 s outages, with $27.5 \%$ in horizontal and $7.4 \%$ under 30 s outages. 
TABLE 3: Average positioning error statistics during three $10 \mathrm{~s}$ signal outages.

\begin{tabular}{lcccc}
\hline Item & Process method & $\mathrm{E}(\mathrm{m})$ & $\mathrm{N}(\mathrm{m})$ & $\mathrm{U}(\mathrm{m})$ \\
\hline \multirow{2}{*}{ RMS } & Prop. & 0.213 & 0.172 & 0.422 \\
\multirow{2}{*}{ Mean } & Conv. & 0.309 & 0.248 & 0.450 \\
& Prop. & -0.082 & 0.001 & 0.133 \\
\multirow{2}{*}{ Max. (abs) } & Conv. & -0.164 & 0.094 & 0.064 \\
& Prop. & 0.883 & 0.685 & 1.573 \\
& Conv. & 0.949 & 0.865 & 1.289 \\
\hline
\end{tabular}

Prop. stands for the proposed method. Conv. stands for the conventional method.

TABLE 4: Average positioning error statistics during three $30 \mathrm{~s}$ signal outages.

\begin{tabular}{lcccc}
\hline Item & Process method & $\mathrm{E}(\mathrm{m})$ & $\mathrm{N}(\mathrm{m})$ & $\mathrm{U}(\mathrm{m})$ \\
\hline \multirow{2}{*}{ RMS } & Prop. & 1.769 & 1.778 & 4.283 \\
& Conv. & 2.254 & 2.144 & 4.627 \\
\multirow{2}{*}{ Mean } & Prop. & -0.871 & 0.701 & 2.357 \\
\multirow{2}{*}{ Max. (abs) } & Conv. & -1.130 & 0.873 & 2.454 \\
& Prop. & 7.708 & 7.099 & 15.167 \\
& Conv. & 8.858 & 8.820 & 16.179 \\
\hline
\end{tabular}

Prop. stands for the proposed method. Conv. stands for the conventional method.

TABle 5: The improvement of accuracy under different outage lengths.

\begin{tabular}{lcc}
\hline Directions & $10 \mathrm{~s}(\%)$ & $30 \mathrm{~s}(\%)$ \\
\hline $\mathrm{E}$ & 31.1 & 21.5 \\
$\mathrm{~N}$ & 30.7 & 17.1 \\
$\mathrm{U}$ & 6.2 & 7.4 \\
Horizontal & 43.6 & 27.5 \\
All & 44.1 & 28.5 \\
\hline
\end{tabular}

By ensuring reliable performance of tightly coupled BDS/ INS integration, the research is expected to achieve the goal of lowering the hardware cost but with a better performance of the whole positioning system. In future work, we will focus on improving tightly coupled integration by introducing differential intersystem biases of GNSS to make the most use of limited available satellites.

\section{Data Availability}

The data used to support the findings of the study are available from the corresponding author upon request.

\section{Conflicts of Interest}

The authors declare that there are no conflicts of interest regarding the publication of this paper.

\section{Authors' Contributions}

Fei Ye conceived and designed the experiments. Shuguo Pan verified the feasibility of the method. Fei Ye and Yunfeng Wang performed the experiments and analyzed the experimental results with Shuguo Pan; Chun Ma participated in algorithm programming. Hao Wang participated in
MATLAB plotting. Fei Ye wrote the paper; BDS singleepoch positioning techniques were carried out under the supervision of Wang Gao.

\section{Acknowledgments}

This work was supported by the National Natural Science Foundation of China (Grant no. 41774027) and the National Key Technologies R\&D Program (Grant no. 2016YFB0502101).

\section{References}

[1] D. Wang, X. Meng, C. Gao, S. Pan, and Q. Chen, "Multipath extraction and mitigation for bridge deformation monitoring using a single-difference model," Advances in Space Research, vol. 60, no. 12, pp. 2882-2895, 2017.

[2] G. Falco, M. Pini, and G. Marucco, "Loose and tight GNSS/ INS integrations: comparison of performance assessed in real urban scenarios," Sensors, vol. 17, no. 2, p. 255, 2017.

[3] J. Wang, D. Liu, W. Jiang, and D. Lu, "Evaluation on loosely and tightly coupled GNSS/INS vehicle navigation system," in Proceedings of the International Conference on Electromagnetics in Advanced Applications (ICEAA), pp. 892-895, IEEE, Queensland, Australia, September 2017.

[4] C. Hide, T. Moor, and M. Smith, "Adaptive Kalman filtering algorithms for integrating GPS and low cost INS," in Proceedings of the Position Location and Navigation Symposium, pp. 227-233, IEEE, Monterey, CA, USA, July 2004.

[5] S. Godha and M. E. Cannon, "GPS/MEMS INS integrated system for navigation in urban areas," GPS Solutions, vol. 11, no. 3, pp. 193-203, 2007.

[6] G. Hu, W. Wang, Y. Zhong, B. Gao, and C. Gu, "A new direct filtering approach to INS/GNSS integration," Aerospace Science and Technology, vol. 77, pp. 755-764, 2018.

[7] G. Hu, L. Ni, B. Gao, X. Zhu, W. Wang, and Y. Zhong, "Model predictive based unscented kalman filter for hypersonic vehicle navigation with INS/GNSS integration," IEEE Access, vol. 8, pp. 4814-4823, 2020.

[8] J. Prieto, S. Mazuelas, and M. Win, "Context-aided inertial navigation via belief condensation," IEEE Transactions on Signal Processing, vol. 1, no. 1, 2016.

[9] F. Wu and Y. Tang, "GPS/INS integrated navigation based on phase smoothing pseudorange," Journal of Geodesy and Geodynamics, vol. 30, no. 1, pp. 130-135, 2013, in Chinese.

[10] A. Angrisano, M. Petovello, and G. Pugliano, "GNSS/INS integration in vehicular urban navigation," in Proceedings of the GNSS10, The Institute of Navigation, Portland, OR, USA, September 2010.

[11] A. Angrisano, M. Petovello, and G. Pugliano, "Benefits of combined GPS/GLONASS with low-cost MEMS IMUs for vehicular urban navigation," Sensors, vol. 12, no. 4, pp. 5134-5158, 2012.

[12] P. Teunissen, "The LAMBDA method for the GNSS compass," Artificial Satellites, vol. 41, no. 3, pp. 89-103, 2006.

[13] R. Monikes, A. Teltschik, J. Wendel et al., "Post-processing GNSS/INS measurements using a tightly coupled fixed-interval smoother performing carrier phase ambiguity resolution," in Proceedings of the Position, Location, and Navigation Symposium, pp. 283-290, IEEE/ION. IEEE, San Diego, CA, USA, April 2006.

[14] K. Badshah and Y. Qin, "Integration and simulations of INS/ GNSS system using the approach of carrier phase 
measurements," IAES International Journal of Robotics and Automation, vol. 4, no. 4, 2015.

[15] Y. Gan and L. Sui, "GNSS/INS integrated navigation using time differenced carrier-phase measurements," Bulletin of Surveying and Mapping, vol. 6, pp. 5-8, 2015, in Chinese.

[16] H. Han, J. Wang, J. Wang, and X. Tan, "Performance analysis on carrier phase-based tightly-coupled GPS/BDS/INS integration in GNSS degraded and denied environments," Sensors, vol. 15, no. 4, pp. 8685-8711, 2015.

[17] S. Liu, F. Sun, L. Zhang, W. Li, and X. Zhu, "Tight integration of ambiguity-fixed PPP and INS: model description and initial results," GPS Solutions, vol. 20, no. 1, pp. 39-49, 2016.

[18] W. Gao, C. Gao, S. Pan, D. Wang, and S. Wang, "Single-epoch positioning method in network RTK with BDS triple-frequency widelane combinations," Acta Geodaetica et Cartographica Sinica, vol. 44, no. 6, pp. 641-648, 2015, in Chinese with English abstract.

[19] J. Li, Y. Yang, H. He, and H. Guo, "An analytical study on the carrier-phase linear combinations for triple-frequency GNSS," Journal of Geodesy, vol. 91, no. 2, pp. 151-166, 2017.

[20] W. Tang, C. Deng, C. Shi, and J. Liu, "Triple-frequency carrier ambiguity resolution for Beidou navigation satellite system," GPS Solutions, vol. 18, no. 3, pp. 335-344, 2014.

[21] W. Gao, C. Gao, S. Pan, G. Yu, and H. Hu, "Method and assessment of BDS triple-frequency ambiguity resolution for long-baseline network RTK," Advances in Space Research, vol. 60 , no. 12, pp. 2520-2532, 2017.

[22] X. He, X. Zhang, L. Tang, and W. Liu, "Instantaneous realtime kinematic decimeter-level positioning with BeiDou triple-frequency signals over medium baselines," Sensors, vol. 16, no. 1, p. 1, 2015.

[23] M. Liu, H. Chai, B. Dong, D. Li, and F. Li, "An algorithm of single-epoch integer ambiguity resolution for reference stations of BDS triple-frequency network RTK," in Proceedings of the 2016 China Satellite Navigation Conference (CSNC), pp. 337-345, Springer, Changsha, China, May 2016.

[24] T. Li, K. Chen, and J. Wang, "Enhanced RTK integer ambiguity resolution with BeiDou triple-frequency observations," in Proceedings of the 2015 China Satellite Navigation Conference (CSNC), pp. 227-238, Springer, Xian, China, May 2015.

[25] Q. Zhao, Z. Dai, Z. Hu, B. Sun, C. Shi, and J. Liu, "Threecarrier ambiguity resolution using the modified TCAR method," GPS Solutions, vol. 19, no. 4, pp. 589-599, 2015.

[26] X. Wang, W. Liu, and G. Sun, "An improved geometry-free three carrier ambiguity resolution method for the BeiDou navigation satellite system," Journal of Navigation, vol. 69, no. 6, pp. 1393-1408, 2016.

[27] Y. Ning and Y. Yuan, "A modified geometry- and ionospheric-free combination for static three-carrier ambiguity resolution," GPS Solutions, vol. 21, no. 4, pp. 1633-1645, 2017.

[28] Y. Tian, D. Zhao, H. Chai, and S. Wang, "BDS ambiguity resolution with the modified TCAR method for medium-long baseline," Advances in Space Research, vol. 59, no. 2, pp. 670-681, 2017.

[29] Y.-F. Yao, J.-X. Gao, J. Wang, H. Hu, and Z.-K. Li, "Real-time cycle-slip detection and repair for BeiDou triple-frequency undifferenced observations," Survey Review, vol. 48, no. 350, pp. 367-375, 2016.

[30] B. Li, "Review of triple-frequency GNSS: ambiguity resolution, benefits and challenges," Journal of Global Positioning Systems, vol. 16, no. 1, 2018.

[31] W. Gao, C. Gao, and S. Pan, "Single-epoch navigation performance with real BDS triple-frequency pseudorange and
EWL/WL observations," Journal of Navigation, vol. 69, no. 6, pp. 1293-1309, 2016.

[32] G. Hu, B. Gao, Y. Zhong, and C. Gu, "Unscented kalman filter with process noise covariance estimation for vehicular INS/ GPS integration system," Information Fusion, vol. 64, pp. 194-204, 2020.

[33] H. Han and J. Wang, "Robust GPS/BDS/INS tightly coupled integration with atmospheric constraints for long-range kinematic positioning," GPS Solutions, vol. 21, no. 3, pp. 1285-1299, 2017.

[34] X. Zhang, F. Zhu, Y. Zhang, F. Mohamed, and W. Zhou, "The improvement in integer ambiguity resolution with INS aiding for kinematic precise point positioning," Journal of Geodesy, vol. 93, no. 7, pp. 993-1010, 2019.

[35] I. Arasaratnam and S. Haykin, "Cubature kalman filters," IEEE Transactions on Automatic Control, vol. 54, no. 6, pp. 1254-1269, 2009. 